\title{
Modeling gene-by-environment interaction in comorbid depression with alcohol use disorders via an integrated bioinformatics
} approach

Richard C McEachin*1,2, Benjamin J Keller ${ }^{2,3}$, Erika FH Saunders ${ }^{1}$ and Melvin G McInnis ${ }^{1,2}$

Address: ${ }^{1}$ Department of Psychiatry, University of Michigan, Ann Arbor, Michigan, USA, ${ }^{2}$ National Center for Integrative Biomedical Informatics, University of Michigan, Ann Arbor, Michigan, USA and ${ }^{3}$ Department of Computer Science, Eastern Michigan University, Ypsilanti, Michigan, USA

Email: Richard C McEachin* - mceachin@umich.edu; Benjamin J Keller - bkeller@emich.edu; Erika FH Saunders - esaunder@umich.edu; Melvin G McInnis - mmcinnis@umich.edu

* Corresponding author

Published: 17 July 2008

BioData Mining 2008, I:2 doi:10.1 I86/I756-038I-I-2

This article is available from: http://www.biodatamining.org/content/l/l/2

(C) 2008 McEachin et al; licensee BioMed Central Ltd.

This is an Open Access article distributed under the terms of the Creative Commons Attribution License (http://creativecommons.org/licenses/by/2.0), which permits unrestricted use, distribution, and reproduction in any medium, provided the original work is properly cited.

\begin{abstract}
Background: Comorbidity of Major Depressive Disorder (depression) and Alcohol Use Disorders (AUD) is well documented. Depression, AUD, and the comorbidity of depression with AUD show evidence of genetic and environmental influences on susceptibility. We used an integrated bioinformatics approach, mining available data in multiple databases, to develop and refine a model of gene-by-environment interaction consistent with this comorbidity.
\end{abstract}

Methods: We established the validity of a genetic model via queries against NCBI databases, identifying and validating TNF (Tumor Necrosis Factor) and MTHFR (Methylenetetrahydrofolate Reductase) as candidate genes. We used the PDG-ACE algorithm (Prioritizing Disease Genes by Analysis of Common Elements) to show that TNF and MTHFR share significant commonality and that this commonality is consistent with a response to environmental exposure to ethanol. Finally, we used MetaCore from GeneGo, Inc. to model a gene-by-environment interaction consistent with the data.

Results: TNF Alpha Converting Enzyme (TACE) activity is suppressed by ethanol exposure, resulting in reduced TNF signaling. TNF binds to TNF receptors, initiating signal transduction pathways that activate MTHFR expression. MTHFR is an essential enzyme in folate metabolism and reduced folate levels are associated with both AUD and depression. Integrating these pieces of information our model shows how excessive alcohol use would be expected to lead to reduced TNF signaling, reduced MTHFR expression, and increased susceptibility to depression.

Conclusion: The proposed model provides a novel hypothesis on the genetic etiology of comorbid depression with AUD, consistent with established clinical and biochemical data. This analysis also provides an example of how an integrated bioinformatics approach can maximize the use of available biomedical data to improve our understanding of complex disease. 


\section{Background}

Comorbidity of Major Depressive Disorder (depression) with Alcohol Use Disorders (AUD) is well documented [1]. Further, significant statistical association between depression and alcohol dependence has long been established [2]. For depressed individuals, the odds ratio for alcohol dependence may be as high 4 to 7 , relative to controls, with a similar increase in prevalence of depression among alcohol dependent individuals $[3,4]$. This association between depression and AUD suggests that there may be one or more common underlying elements influencing susceptibility to both disorders.

Depression, AUD, and the comorbidity of depression with AUD are complex traits, demonstrating both genetic and environmental influences on susceptibility. Depression is familial, with estimates of heritability as high as $54 \%$ [5]. AUD is also familial, with heritability estimates in the range of 50 to $60 \%$ [6]. Comorbidity of depression with AUD also shows evidence of both genetic and shared environmental influences [7-10]. Given evidence of association between depression and AUD, as well as evidence that both the individual traits and the comorbidity are influenced by both genetic and environmental factors, we used an integrated bioinformatics approach, maximizing the use of available data in multiple databases, to investigate the hypothesis that interacting genetic and environmental influences are common underlying elements of susceptibility to comorbid depression with AUD. We report the development and testing of a model of this comorbidity, as well as implications for future research into comorbid depression with AUD.

\section{Methods \\ Queries against NCBI Databases}

In this analysis, we used available data in NCBI databases, plus three software suites (DAVID, PDG-ACE, and GeneGo). To identify potential candidate genes, we queried the Entrez Gene database [11] for "depression AND ethanol AND human [orgn]". Since Entrez Gene surveys the whole human genome, this approach is complementary to Whole Genome Association (WGA) analysis [1214]. WGA surveys many genetic variants (e.g. 1 million Single Nucleotide Polymorphisms (SNPs)) across the genome, while this text-based query surveys all genes in Entrez Gene. WGA provides indirect information on potential associations of genes with disease by associating nearby SNP variants with disease, while Entrez Gene queries associate genes with disease based on curated text describing the genes. Each approach has advantages and disadvantages, highlighted in Table 1, though we believe that the query-based approach offers significant advantages in the initial phase of candidate gene analysis. First, in recent years Entrez Gene has become a rich source of information on many genes, while WGA typically focuses on a very limited information source (e.g. SNP association). Second, data provided by NCBI are free to the users. While some of the genotypic and phenotypic data required for WGA are becoming available in free public repositories, these data are generally expensive to acquire. Third, data derived from curated sources such as Entrez Gene are backed by multiple rounds of experimentation and peer review, so the believability of a positive result, as measured by Positive Predictive Value [PPV = True Positives/(True positives + False Positives)], is very high. Given these strengths, a query based approach should be considered for the initial phase of candidate gene analysis, which

Table I: Comparison of Whole Genome Association (WGA) to text based, Entrez Gene, search for candidate genes.

\begin{tabular}{|c|c|c|}
\hline & \multicolumn{2}{|c|}{ Identifying Candidate Genes } \\
\hline & WGA & Text Based Search \\
\hline Data Source & Genotyping and Phenotyping & Entrez Gene \\
\hline Current Availability of Data & Limited & Available \\
\hline Expense & High & Free (Taxpayer Subsidized) \\
\hline Sensitivity & Low; limited number of markers tested & Low; much remains unknown about most genes \\
\hline Positive Predictive Value & Low; many false positives & $\begin{array}{l}\text { High; curated information is backed by experimental } \\
\text { data and peer review }\end{array}$ \\
\hline Population Specific & Yes & $\begin{array}{l}\text { Generally no, although population specific information } \\
\text { may be included in some records }\end{array}$ \\
\hline Specific Variants Identified & $\begin{array}{l}\text { Yes, although SNPs that tag haplotypes may not } \\
\text { be disease causing variants }\end{array}$ & $\begin{array}{l}\text { Generally no, although specific variants may be } \\
\text { annotated. Separate genotyping is usually required for } \\
\text { validated candidates }\end{array}$ \\
\hline Multi-Gene Effects on Phenotype & Limited & Yes \\
\hline Complex Phenotypes & Limited & Yes \\
\hline
\end{tabular}

Text based searches, using data at $\mathrm{NCBI}$ as the first source for candidate gene data, provide advantages in data availability, price, and positive predictive value. 
will then maximize the value of subsequent association testing.

The Entrez Gene database provides reliable information about most genes, but the Entrez Gene record is a summary, so the information it provides is necessarily limited. Therefore, we queried a second NCBI database, PubMed [15], for more complete information on a variety of hypotheses developed in this analysis. Table 2 shows the short form of each PubMed query in column 1 and the citation count is in column 2. For each PubMed query, the actual query was qualified by Medical Subject Headings $(\mathrm{MeSH})$ annotation [16] and the detailed queries are included in Additional file 1. Publications are annotated by MeSH terminology to provide a consistent way to retrieve information in publications that may use different terminology for the same concepts. In addition, querying PubMed by MeSH annotation yields a high likelihood that publications returned by the query refer to the queried terms in the appropriate context (high PPV). For example, in looking for association between depression and AUD, we queried PubMed for "( "Depressive Disorder" [Mesh] OR "Depression" [Mesh] OR "Depressive Disorder, Major" [Mesh]) AND ("Ethanol" [Mesh] OR "Alcohol-Induced Disorders, Nervous System" [Mesh])". The resulting 263 citations returned are consistent with the statistically significant association between depression and AUD found by Hasin and Glick [2]. Using the University of Michigan's electronic journals library, we made an exhaustive review the 263 citations returned by this query

Table 2: PubMed queries and citation counts

\begin{tabular}{ll}
\hline Short form, PubMed Queries & PubMed Citation Count \\
\hline depression AND ethanol & 263 \\
depression AND MTHFR & 12 \\
ethanol AND MTHFR & 5 \\
depression AND ethanol AND MTHFR & 0 \\
depression AND TNF & 92 \\
ethanol AND TNF & 321 \\
depression AND ethanol AND TNF & 1 \\
depression AND NFkB & 2 \\
ethanol AND NFkB & 185 \\
depression AND ethanol AND NFkB & 0 \\
depression AND TNFR & 19 \\
ethanol AND TNFR & 44 \\
depression AND ethanol AND TNFR & 0 \\
depression and APOE & 91 \\
ethanol and APOE & 42 \\
depression AND ethanol AND APOE & 0 \\
\hline
\end{tabular}

PubMed queries were used to search for evidence consistent with various hypotheses on 9 December, 2007. Queries were qualified by Medical Subject Headings (MeSH) annotation to minimize spurious associations. The actual queries, including MeSH qualifications, are provided as Additional file I. Column I has a short form of the query and column 2 shows the number of citations that meet the query criteria. to quantify the PPV of this MeSH qualified PubMed query. In this review, we looked for clear evidence that the authors were studying AUD and depression, in English language publications, either full text or abstracts. We were able to unequivocally assess the accuracy of MeSH annotation for AUD and depression in 179 citations. Of these 179 publications, 171 clearly refer to both AUD and depression, though not necessarily the comorbidity of AUD with depression. Of the eight remaining publications, five are correctly annotated for ethanol, but ethanol is used as an important reagent in the experimental procedures. The final three publications are incorrectly annotated for ethanol, though they refer to pharmacologically related chemicals (flupenthixol, haloperidol, and pirbuterol). We consider these eight publications to be false positives with respect to our original query, yielding a 95.5\% (171/179) PPV for our query.

There are, however, limitations to the use of MeSH annotation in querying publications. First, while MeSH contains thousands of Medical Subject Headings, it is not an exhaustive annotation of all potential biomedical contexts. Second, there may be missing annotation or ambiguity in the annotation for some publications. For example, as mentioned in the PPV analysis, not all of the 171 papers that we counted as True Positives were specifically about comorbid AUD with depression. The earlier papers tended to refer to AUD and depression as co-occurring phenotypes, while the later papers focused on evaluating the comorbidity. There also may be ambiguity in the user's choice of MeSH terms queried, and some publications may report negative associations, though the bias against publication of negative results minimizes the number of these papers. And finally, a lack of citations is not evidence of a lack of association. While co-occurrence of queried terms is not proof of association, co-occurrence does provide a quantitative measure of peer reviewed research on the association of these specific terms and complements other forms of data that can be mined. In future analyses, Natural Language Processing will help to improve these queries by mining the full text of publications for a wide variety of concepts, and provide additional evidence of association among terms. Given believable candidate genes, derived from queries of available data at Entrez Gene and PubMed, we continued the analysis using DAVID, PDG-ACE, and MetaCore (GeneGo, Inc.) to understand how these candidate genes may be related to the etiology of AUD, depression, and comorbid AUD with depression.

\section{DAVID, PDG-ACE, and GeneGo}

DAVID software, the PDG-ACE algorithm, and the GeneGo suite perform related tasks. The essential assumption in the use of all three tools is that, in complex diseases, multiple genetic influences converge on a single 
phenotype. The three tools use different approaches to identify how multiple genes may influence the phenotype of interest and assess the statistical significance of the results seen.

DAVID [17] uses a database of available gene annotation to overlay candidate genes onto predefined gene sets. For example, genes may be organized into predefined sets based on annotation for their participation in a Gene Ontology function, process, or component [18]. A set of candidate genes is then derived from a genotype/phenotype analysis (e.g. WGA, microarray expression, or textbased database query) and the candidate genes are overlaid on the predefined gene sets. If a large proportion of the candidate genes are found to be annotated for a given gene set (e.g. an over-represented Gene Ontology process), the candidate genes may have an important impact on the process represented by that gene set. Since the candidate genes were initially derived from a genotype/phenotype relationship, the over-represented process may be important in the phenotype [19].

However, since much gene annotation remains incomplete, PDG-ACE [20] [see Additional file 2] serves as an adjunct to software that depends on gene annotation. As noted, the Entrez Gene database offers a reliable summary of information on a wide range of genetic and environmental influences for each gene (e.g. gene function, disease influences, localization, environmental effects). However, much of the information available in Entrez Gene is free text, rather than formal annotation, limiting the use of tools like DAVID. We created the PDG-ACE algorithm to overcome this limitation by mining Entrez Gene text to identify biomedical keywords that are common and significantly over-represented in the descriptions of genes at a selected locus pair, relative to all locus pairs in the genome.

The PDG-ACE algorithm uses a controlled vocabulary of biomedical keywords to limit the search to concepts likely to be useful in understanding disease etiology and show statistically significant over-representation. For a given locus pair, PDG-ACE mines the Entrez Gene text to find keywords from the controlled vocabulary that are common across the locus pair. For keywords that are common across the locus pair, significance of over-representation is established by permutation testing. Keywords that are common and significantly over-represented across the locus pair highlight rare combinations of genes that share the biomedical concepts associated with the keywords. Testing of the PDG-ACE algorithm, using both positive controls (documented gene-gene interactions in complex disease) and negative controls (randomly selected gene pairs), demonstrates that PDG-ACE provides insight into the etiology of true genetic interactions, while excluding loci where the data is insufficient to identify interactions [20]. As with DAVID, the loci are initially defined by the disease phenotype, so the over-represented keywords may offer insight into the nature of a genetic interaction in disease etiology. In applying the PDG-ACE algorithm, we define genetic interaction to mean a "statistically significant multi-gene or multi-gene-by-environment influence on the phenotype", consistent with Hartman et al. [21]. This definition is deliberately broad, allowing PDG-ACE to identify unannotated influences on the phenotype, ranging from well-defined influences (e.g. epistasis, protein-protein binding, canalization, genetic robustness, buffering) to completely novel influences, as long as the influence is multi-gene (or multi-gene-by-environment) and statistically significant. The complete list of keywords used in this analysis is included in Additional file 3. Software implementing the PDG-ACE algorithm is available on request from Dr. Ben Keller, bkeller@emich.edu.

The GeneGo suite [22] uses the MetaCore database of documented gene-gene (protein-protein) interactions [23], as well as a set of graphical and statistical tools, to allow researchers to build gene networks based on relationships among genes. The MetaCore interactive database has been manually curated from publications describing interactions among proteins and small molecules of biological relevance in humans. We used GeneGo to place our candidate genes into a cellular context, evaluate the significance of gene networks that our candidates participate in, and build a model that integrates all of the information derived in our analysis. Specifics of the parameter settings used in the GeneGo analysis are included in Table 3. Notably, we accepted only direct interactions, curated by human reviewers.

\section{Results}

Based on prior evidence consistent with genetic and environmental influences on susceptibility to comorbid depression with AUD, we started the analysis with the hypothesis that genetic influences participate in depression and AUD. Figure 1 shows the analysis flow, including the initial hypothesis, three rounds of hypothesis testing and generation, and model building based on the results of our testing.

\section{Genetic Influences on Comorbidity}

We first searched for evidence of genetic influences on depression and AUD by querying NCBI's Entrez Gene database for "depression AND ethanol AND human [orgn]". This query returned exactly three genes: Tumor Necrosis Factor (TNF superfamily, member 2, GeneID 7124), Methylenetetrahydrofolate Reductase (MTHFR, 5,10-methylenetetrahydrofolate reductase (NADPH), GeneID 4524) [24-29], and APOE (apolipoprotein E, GeneID 348). However, the Gene Ontology annotation 
Table 3: Parameter settings used in GeneGo analysis

\begin{tabular}{ll}
\hline Options & Setting \\
\hline Use canonical pathways & Yes \\
Network type & Merged \\
Build options & Analyze network (transcription factors) \\
\hline Advanced options & \\
\hline Discard objects user list & \\
Use indirect interactions & No \\
Use interactions weights & Discard \\
Add complementary objects & No \\
Filter interactions by confidence level & Yes \\
Use binding interactions (special cases) & Curated only \\
Group networks & Discard \\
Discard objects experiments & No \\
Use unspecified reactions & No \\
\hline
\end{tabular}

GeneGo analysis was performed with MetaCore ${ }^{\mathrm{TM}}$ version 4.5, build I I I65, on 26 November 2007. TNF and MTHFR were the input gene set.

for APOE, "response to ethanol", is inferred from electronic annotation, rather than manual curation. In an effort to use only the most reliable information to establish our initial candidate genes, we excluded APOE from the formal analysis. However, we have included a brief analysis of $A P O E$ in the results.

We searched PubMed for supporting information on potential roles of the proteins coded by TNF and MTHFR in depression, AUD, and comorbid depression with AUD. In searching PubMed, we found evidence that both TNF and MTHFR have been associated with depression and with ethanol in the literature, but not with the comorbidity of depression with AUD (Table 2). Notably, the result in Table 2 showing that TNF is associated with both depression and ethanol in a single publication is one of the false positive results identified in the PPV analysis. We didn't find evidence of MTHFR being associated with both depression and AUD in any single paper.

In total, the published literature is consistent with the hypothesis that TNF and MTHFR may both influence, or be influenced by, depression and AUD. We consider TNF and MTHFR to be valid candidate genes for both depression and AUD. Given these candidate genes, we next sought evidence that they participate in the comorbidity via genetic interaction.

\section{Interaction between TNF and MTHFR via DAVID and PDG-ACE}

Given evidence of genetic influences on depression and AUD, we refined the hypothesis to include interaction between TNF and MTHFR. We first used DAVID software to explore functional annotation consistent with interaction between TNF and MTHFR. DAVID looks for over-rep- resentation of candidate genes in signaling or metabolic pathways, Gene Ontology processes, etc., based on available gene annotation. TNF and MTHFR were not found to be significantly over-represented in any gene set tested by DAVID.

Since most genes are only partially annotated, we next explored potential interactions between TNF and MTHFR via the PDG-ACE algorithm [20]. A total of 149 keywords were found to be common across the TNF/MTHFR gene pair, out of 2,531 keywords in this controlled vocabulary. We checked these 149 common keywords for over-representation using 10 million iterations in permutation testing. After a Bonferroni correction for 2,531 hypothesis tests, 27 keywords were found to be significantly over-represented (p-value < 0.05) in the TNF/MTHFR gene pair, relative to randomly selected gene pairs in the human genome. Six of these 27 keywords were found to be used in different contexts in the TNF and MTHFR Entrez Gene records and were eliminated; leaving 21 keywords that are common and significantly over-represented across the TNF/MTHFR gene pair (Table 4). Given this evidence of interaction between TNF and MTHFR in AUD and depression, and consistent with expected environmental influences on complex diseases, we further refined the hypothesis to include gene-by-environment interaction influencing the comorbidity.

\section{Environmental Influence of Ethanol Exposure}

Each significantly over-represented keyword identified by PDG-ACE represents one hypothesis on the etiology of a genetic interaction between TNF and MTHFR in depression and AUD. We tested each of these hypotheses by querying PubMed for "depression AND ethanol AND keyword" (Table 4). As in the previous round of testing, we 


\section{Prior Evidence: Comorbidity and Familiality of Depression with AUD}

Hypothesis: One or more genetic

Influence(s) on Comorbidity

Candidate genes identified and

validated via $\mathrm{NCBI}$ database queries

ANALYSIS FLOW

Hypothesis: Genetic Interaction

Between TNF and MTHFR

Evidence consistent with hypothesis

via PDG-ACE algorithm

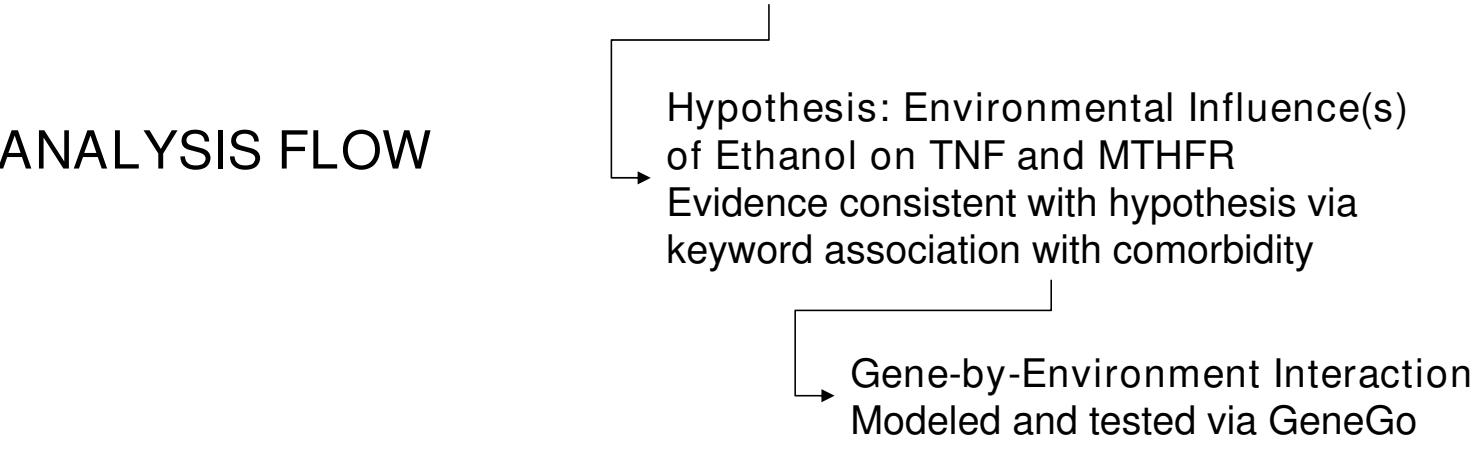

Figure I

Analysis Flow. Analysis started with prior evidence of potential genetic and environmental influences on comorbidity, followed by testing for genetic influences, genetic interactions, and environmental influences. The resulting model of gene-by-environment interaction is consistent with clinical and biochemical data.

used MeSH annotation to limit the queries and we used citation counts to provide indications of relevant association in the literature. Notably, since ethanol is a keyword in the PDG-ACE controlled vocabulary that we used, it acts as a positive control in this analysis and is the top keyword when ranked by relevance. In addition to ethanol, the top keywords ranked by relevance to depression and AUD are "consumption", "background", and "intake". In the Entrez Gene records for TNF and MTHFR, intake and consumption both refer to intake of alcohol, while background refers specifically to genetic background. Results of the PDG-ACE analysis are consistent with the hypothesis that genetic background, via TNF and MTHFR, as well as environmental influences, via alcohol intake or consumption, are interacting elements of susceptibility in comorbid depression with AUD. Given evidence of genetic interaction between TNF and MTHFR, as well as gene-by-environment interaction in comorbid depression with AUD, we next sought to understand TNF and MTHFR in a cellular context.

\section{Model Building via GeneGo}

We used the GeneGo suite $[22,23]$ to place TNF and MTHFR in a cellular context. Results of the PDG-ACE analysis suggested that ethanol consumption exerts an environmental influence on this genetic system. Also, TNF is a secreted protein that responds to the environment by binding TNF receptors to influence intracellular signal transduction pathways that regulate gene expression. Given these inputs, we focused our GeneGo analysis on cell signaling via signal transduction and the regulation of gene expression within the cell (input parameters in Table 3).

The most significant network found by GeneGo (Figure 2) shows a feedback loop among TNF (a.k.a. TNF-alpha), TNF-R1 (a.k.a. TNFRSF1A, GeneID 7132), and NF-kB (a.k.a. NFKB1, GeneID 4790). Note that these proteins do not act in isolation, but the GeneGo graphic shows the essential elements of the network based on annotation in GeneGo's MetaCore database. In the network identified by GeneGo, conditions in the extracellular environment 
Table 4: Significant biomedical keywords identified by PDG-ACE across the TNF/MTHFR gene pair, and relevance to depression with AUD

\begin{tabular}{ll}
\hline Keyword & \# PubMed citations for query: \\
\hline Ethanol & 263 \\
Consumption & 29 \\
Background & 12 \\
Intake & 10 \\
Child & 9 \\
Mortality & 6 \\
Transplant & 2 \\
Mineral & 1 \\
Hemodialysis & 0 \\
Abdominal & 0 \\
Carotid & 0 \\
Wall & 0 \\
Preoperative & 0 \\
Lumbar & 0 \\
kidney transplantation & 0 \\
Spine & 0 \\
Homozygote & 0 \\
Mediterranean & 0 \\
hormone replacement therapy & 0 \\
p53 gene & 0 \\
Sickle & 0 \\
\hline
\end{tabular}

Each keyword derived from the PDG-ACE analysis (column I) was queried against the PubMed database via the query: ("Depressive Disorder" [Mesh] OR "Depression" [Mesh] OR "Depressive Disorder, Major" [Mesh]) AND ("Ethanol" [Mesh] OR "AlcoholInduced Disorders, Nervous System" [Mesh]) AND Keyword. Citation counts are used as an indication of the keyword's relevance in comorbid depression with AUD.

are sensed inside the cell via binding of TNF to TNF-R1, activating TNF-R1 $[30,31]$. Activated TNF-R1 activates NF$\mathrm{kB}[32,33]$, which subsequently activates the expression of both TNF [34] and MTHFR [35,36]. Given the potential for TNF-R1 and NF-kB to impact this network, we queried PubMed for potential influences of these genes on depression and AUD (Table 2). We found citations consistent with roles for both of these genes in depression and AUD.

Genetic variation or extracellular signals that affect any part of the feedback cycle (Figure 2) would tend to be amplified over a number of cycles, potentially leading to a growing imbalance in the system over time. In this network, NF-kB activates the expression of MTHFR, which metabolizes folate, so imbalances in the system would be expected to lead to imbalances in folate metabolism. This result is consistent with evidence of decreased folate levels in alcoholism [37]. In addition, reduced folate levels are associated with depression [38], where research on etiology suggests that reduced folate levels may alter neurotransmitter metabolism [39-41].

Summarizing our results to this point, we found evidence that both TNF and MTHFR are associated with both depression and alcohol in the literature, TNF and MTHFR interact to influence the comorbidity and alcohol exerts an environmental influence on this genetic network, and folate levels are altered in both depression and alcohol dependence. Investigating the environmental influence of ethanol on TNF, we found that ethanol intake suppresses the function of TNF Alpha Converting Enzyme (TACE, a.k.a. ADAM17, GeneID 6868) [28], leading to reduced TNF signaling. Integrating these lines of evidence, we propose a model of comorbid depression with AUD (Figure $3)$. In this model, environmental ethanol exposure suppresses TNF signaling by inhibiting TACE [28]. Reduced TNF signaling reduces TNF-R1 activation [30,31], which then reduces NF-kB activation [32]. NF-kB activates the expression of both TNF $[33,34]$ and MTHFR $[35,36]$, so reduced NF-kB activation contributes to further reduction in TNF signaling as well as reduced MTHFR expression. Reduced expression of MTHFR would be expected to alter folate metabolism and increase susceptibility to depression [38].

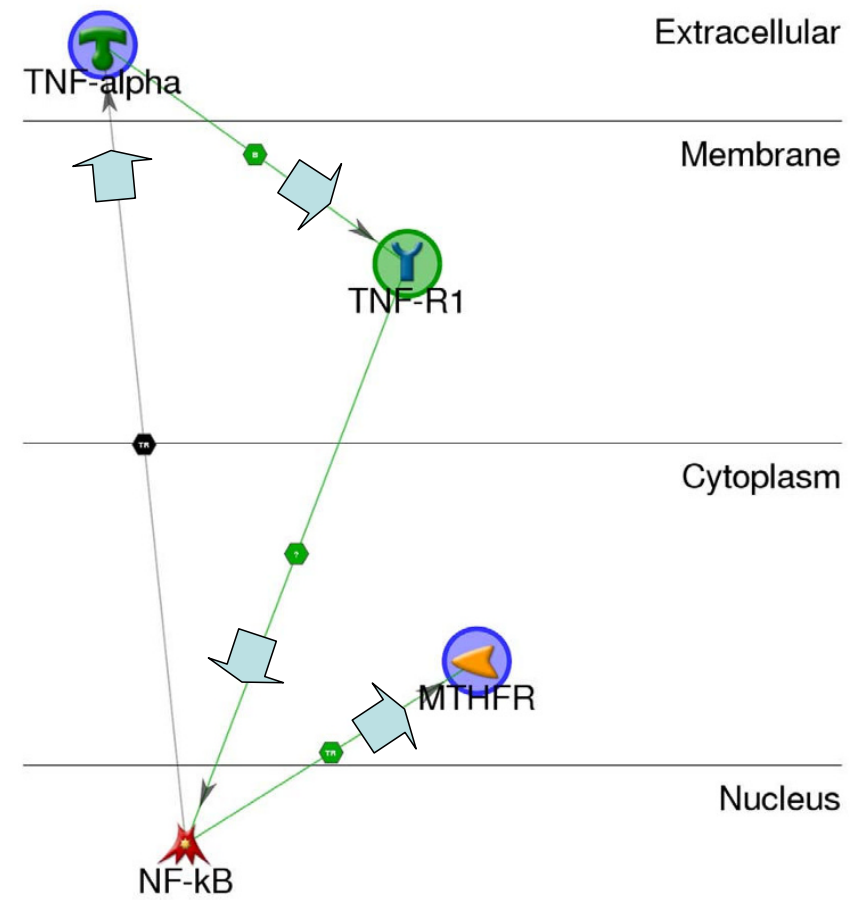

Figure 2

Top scoring GeneGo network including TNF and MTHFR. This four-gene network is the best fit found by GeneGo (p-value 3.45e-08) for TNF and MTHFR. TNF

(TNF-alpha) activates TNFR (TNF-RI), which then activates NF-kB. NF-kB regulates the expression of both MTHFR and TNF, creating a feedback loop among TNF, TNFR, and NF$\mathrm{kB}$. 


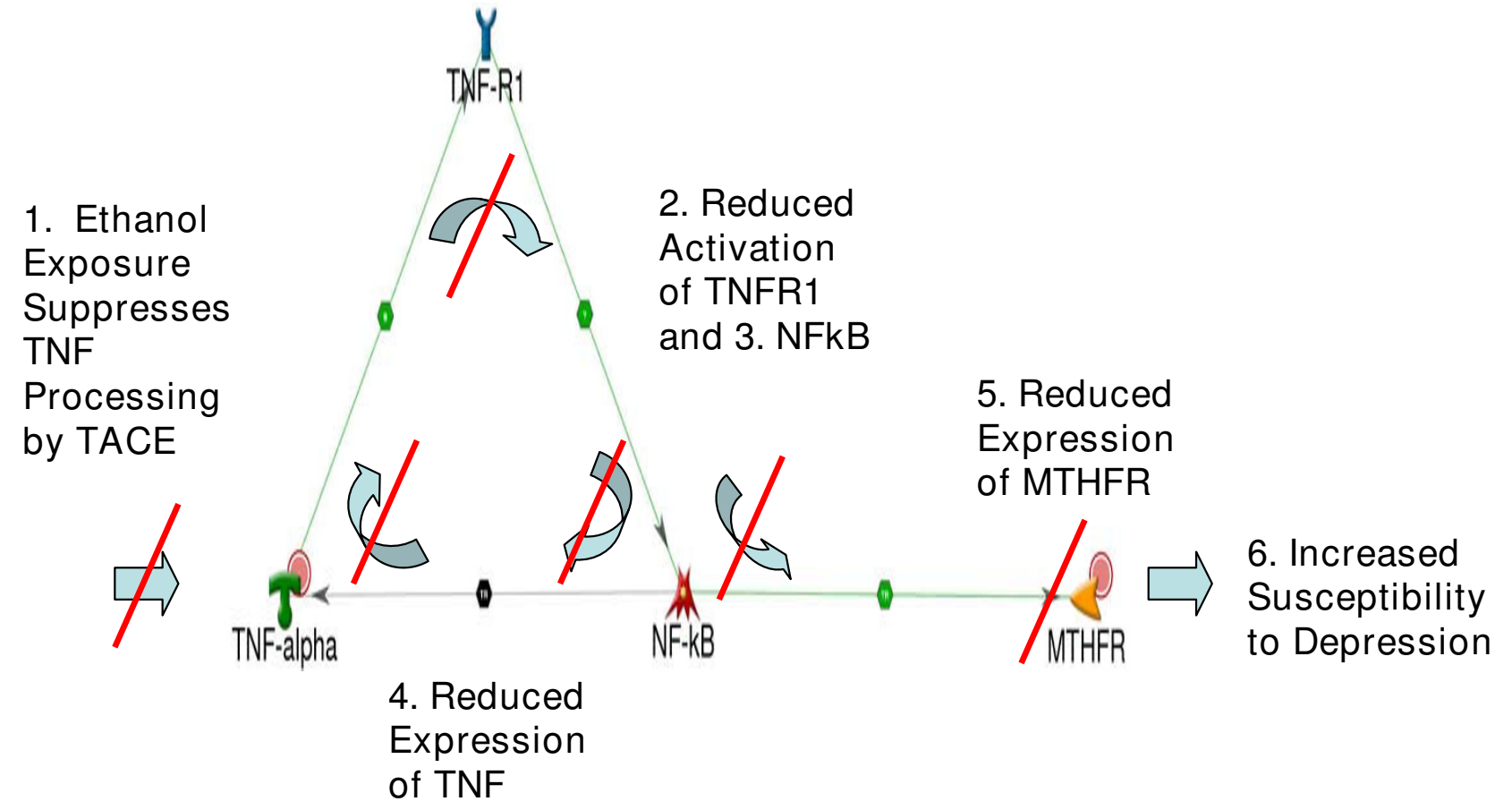

Figure 3

Proposed Model of Comorbid Depression with AUD. This model, incorporating evidence gathered in hypothesis testing, and consistent with an environmental effect of ethanol on the genetic network, shows how ethanol consumption would be expected to result in decreased folate metabolism and increased susceptibility to depression for genetically susceptible individuals.

Signal transduction, leading to transcriptional regulation of both TNF and MTHFR, is an essential assumption of this model. In addition to building networks, GeneGo analyzes networks for over-representation of genes that participate in Gene Ontology (GO) processes (similar to the DAVID analysis described), then ranks the GO proc- esses by p-value. Table 5 shows that "positive regulation of transcription from RNA polymerase II promoter" is the most significantly over-represented GO process in this network. This result is consistent with the hypothesis that environmental influences, via ethanol exposure, are transduced through this genetic network to alter TNF and

Table 5: Ranking of Gene Ontology processes for GeneGo network in Figure 2

\begin{tabular}{ll}
\hline Gene Ontology Process & P-value \\
\hline positive regulation of transcription from RNA polymerase II promoter & $3.8 \mathrm{E}-06$ \\
negative regulation of interleukin-I2 biosynthetic process & $5.3 \mathrm{E}-06$ \\
inflammatory response & $9.5 \mathrm{E}-06$ \\
positive regulation of I-kappaB kinase/NF-kappaB cascade & $1.5 \mathrm{E}-05$ \\
positive regulation of transcription, DNA-dependent & $1.5 \mathrm{E}-05$ \\
regulation of I-kappaB kinase/NF-kappaB cascade & $1.8 \mathrm{E}-05$ \\
positive regulation of transcription & $2.9 \mathrm{E}-05$ \\
positive regulation of nucleobase, nucleoside, nucleotide and nucleic acid metabolic process & $3.3 \mathrm{E}-05$ \\
regulation of interleukin-I2 biosynthetic process & $3.8 \mathrm{E}-05$ \\
interleukin-I 2 biosynthetic process & $3.8 \mathrm{E}-05$ \\
response to wounding & $3.9 \mathrm{E}-05$ \\
interleukin-I 12 production & $4.8 \mathrm{E}-05$ \\
\hline
\end{tabular}

Regulation of transcription is the most significantly over-represented Gene Ontology process for this network, consistent with the hypothesis that environmental influences, via ethanol exposure, are transduced through this genetic network to alter TNF and MTHFR expression, folate metabolism, and susceptibility to depression. 
MTHFR expression, folate metabolism, and susceptibility to depression.

Apolipoprotein E (APOE) was excluded from the formal analysis because the annotation in Entrez Gene was inferred, rather than curated. In pursuing the hypothesis that APOE is involved in comorbid depression with AUD, we re-started the analysis including APOE. We queried PubMed for citations consistent with this hypothesis (Table 2) and found evidence of APOE's role in both depression and AUD, but no evidence for a role in the comorbidity. PDG-ACE analysis did not produce any significant results after correction for multiple hypothesis tests. We attempted to insert APOE into the GeneGo network (Figure 2) and found no annotated interactions between APOE and the other genes in the network. Apolipoprotein E may well have an influence on comorbid depression with AUD but, if so, the mechanism is either independent of the network modeled or, more likely, the data is not yet available to reliably connect APOE to this network.

\section{Discussion}

Comorbid depression with AUD shows evidence of both genetic and environmental influences on susceptibility. In three phases of hypothesis generation and testing (NCBI database queries, PDG-ACE and GeneGo analyses) we established and tested a model of gene-by-environment interaction that shows evidence of influencing the comorbidity and is consistent with established knowledge about AUD and depression [37]. We first hypothesized that common genetic influences affect AUD and depression. We tested this hypothesis by searching for candidate genes and found published evidence, via Entrez Gene and PubMed, supporting the roles of TNF and MTHFR in depression and AUD. Given evidence of a multi-gene influence on the comorbidity, we hypothesized that TNF and MTHR participate in a genetic interaction influencing the comorbidity. Mining the Entrez Gene records for TNF and MTHFR via the PDG-ACE algorithm, we found twenty one keywords that are common and significantly overrepresented across the gene pair, consistent with interaction between these genes in comorbid AUD with depression. In addition, among the significant keywords, those that are most often associated with depression and AUD in the literature are suggestive of an environmental effect on this genetic interaction via ethanol intake or consumption. Given evidence that TNF and MTHFR participate in a genetic interaction that may be influenced by environmental exposure to ethanol, as well as evidence that TNF influences signal transduction pathways in response to the environment, we hypothesized signal transduction as the most appropriate model of the gene-by-environment interaction. Modeling this hypothesis via GeneGo, we found that both TNF and MTHFR are influenced by a genetic feedback cycle that incorporates environmental ethanol exposure into folate metabolism. Altered folate levels, as well as AUD, are consistently linked to depression [42-44].

Mason and Choi [37] review other mechanisms (decreased dietary intake of folate, decreased intestinal absorption, increased urinary secretion, and cleavage of the folate molecule) that have been purported to reduce the bio-availability of folate with excessive ethanol intake. In addition, they review the adverse effects that ethanol can have on one-carbon metabolism, a process that includes the synthesis of folate. Mason and Choi show five enzymes involved in one-carbon metabolism including MTHFR, Cystathionine beta-synthase (CBS, GeneID 875), Betaine Homocysteine Methyltransferase (BHMT, GeneID 635), Serine Hydroxymethyltransferase 1 (SHMT, GeneID 6470), and Methionine Synthase (MTR, GeneID 4548). Figure 4 is a GeneGo graphic showing how TNF signaling may impact every one of these enzymes by regulating their expression. This network, still fairly simple, includes all of the elements of the proposed model (Figure 2) and provides a suggestion of how AUD impacts one-carbon metabolism in a complex genotype-phenotype relationship. Arguably, because all of the enzymes seen in this network affect folate metabolism, they all are candidates for influencing comorbid depression with AUD.

Based on the relationship between folate metabolism and one-carbon metabolism, we searched for evidence that the one-carbon metabolic process influences depression, AUD, and the comorbidity. Table 6 shows evidence that several of these genes may have influences on depression or AUD. The larger hypotheses, depression AND folate, ethanol AND folate, show 133 and 237 citations, respectively, though we did not find evidence for depression AND ethanol AND folate. In Figure 4, two additional genes, p53 (TP53, GeneID 7157) and HNF4a (HNF4alpha, GeneID 3172), are shown to participate in the network. Searching for evidence of these genes in depression, AUD, and the comorbidity, we found evidence for association between p53 and ethanol (Table 6). Interestingly, "p53 gene" is one of the 21 keywords that we found to be common and significantly overrepresented across the TNF/MTHFR gene pair in the PDG-ACE analysis (Table 4).

Notably, while the evidence assembled in this analysis is consistent with our hypothesized model of gene-by-environment interaction of comorbid depression with AUD, much data remains missing. For example, we emphasize the environmental influence of alcohol consumption, though we have little information on how alcohol dosage or the duration of alcohol exposure might affect genetic influences in our model. Equally, while we have identified 


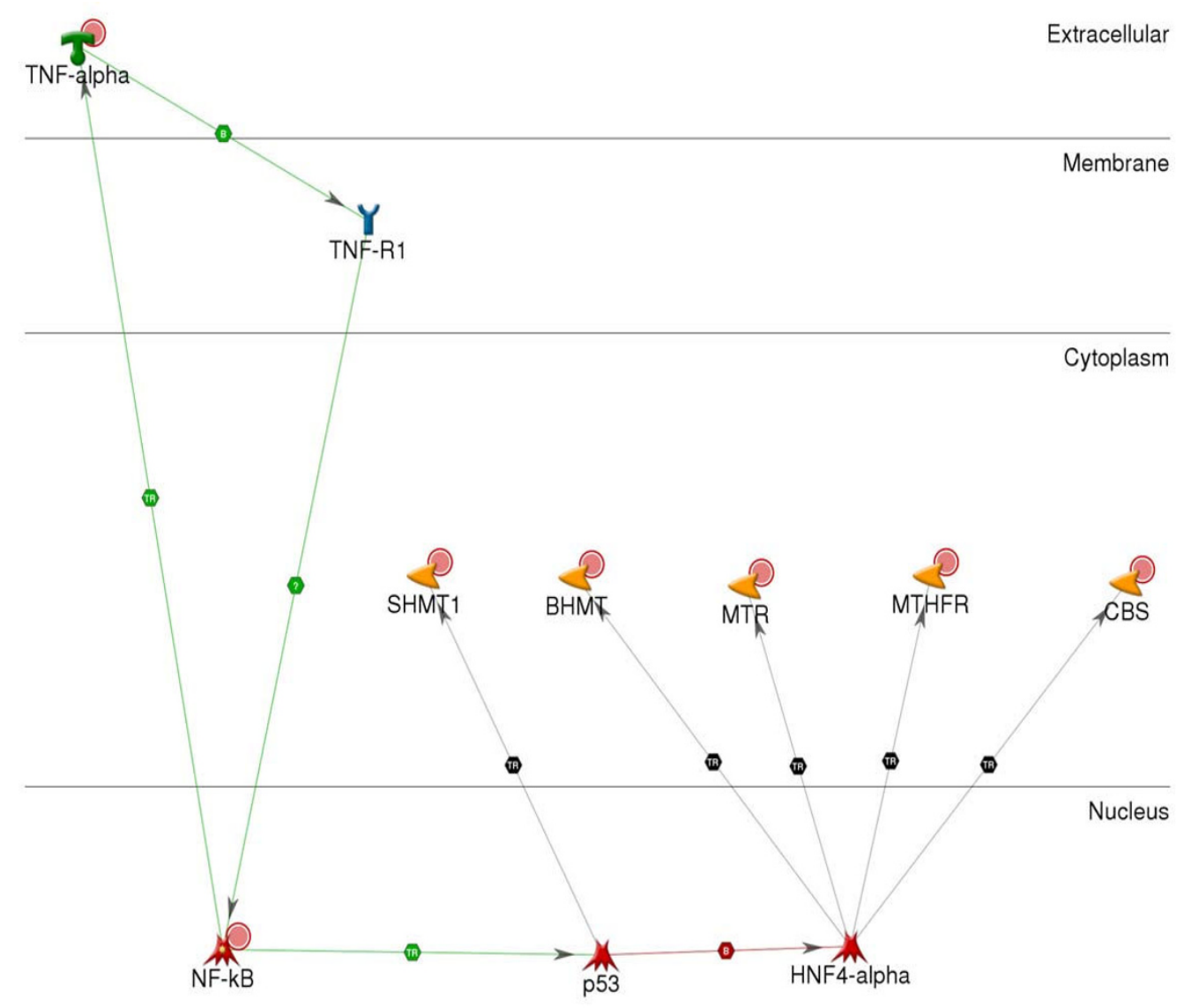

\section{Figure 4}

GeneGo graphic illustrating how TNF signaling impacts one-carbon metabolism. Folate metabolism is one element of one-carbon metabolism and TNF signaling influences all of the enzymes involved. As a result, variations in any of the genes involved in one-carbon metabolism could influence folate metabolism and susceptibility to depression.

a network of genes that may influence the comorbidity, we have limited information on heritable variation that would be expected to increase or decrease susceptibility. To date, the C677T variant of MTHFR is associated with both susceptibility to depression $[27,45,46]$ and ethanol response [26], and levels of TNF mRNA have been associated with depression [25]. Cytogenetic band 6p21, the chromosomal location of TNF, has recently been associated with chromosomal aberrations in alcoholism [47]. However, the genetic influences of this network are likely to be much more complex than what we know so far. Missing data on these influences await follow-on analyses (e.g., targeted genotyping in an affected population versus controls, animal modelling) that can be informed by the model developed here. Also, given the model proposed, future data from WGA or microarray studies can be tested on a reduced number of hypotheses, thus increasing the power of these tools.

\section{Implications}

Implications of this model are consistent with dietary guidance recommending monitoring and appropriate supplementation of folate for patients in treatment for depression [48-50], AUD, or comorbid depression with AUD [37,38,42-44]. In addition, identification of the variants associated with risk could be useful in prognosis and treatment of either or both conditions [51]. Pharmacogenomic approaches are being successfully implemented in psychiatry, to the great benefit of patients with specific genetic variants [52-54], and the identification of disease predisposing variants will likely improve the prognosis for depression with AUD.

\section{Conclusion}

The model developed in this analysis represents one mechanism whereby, for genetically susceptible individuals, alcohol intake could lead to altered folate metabolism and increased susceptibility to depression. The effect of 
Table 6: Additional hypotheses tested based on GeneGo modelling

\begin{tabular}{ll}
\hline Short form, PubMed Queries & \# PubMed Citations \\
\hline depression AND CBS & 1 \\
ethanol AND CBS & 2 \\
depression AND ethanol AND CBS & 0 \\
depression AND BHMT & 0 \\
ethanol AND BHMT & 6 \\
depression AND ethanol AND BHMT & 0 \\
depression AND SHMT & 0 \\
ethanol AND SHMT & 0 \\
depression AND ethanol AND SHMT & 0 \\
depression AND MTR & 1 \\
ethanol AND MTR & 17 \\
depression AND ethanol AND MTR & 0 \\
depression AND folate & 133 \\
ethanol AND folate & 237 \\
depression AND ethanol AND folate & 0 \\
depression AND p53 & 0 \\
ethanol AND p53 & 43 \\
depression AND ethanol AND p53 & 0 \\
depression AND HNF4a & 0 \\
ethanol AND HNF4a & 0 \\
depression AND ethanol AND HNF4a & 0 \\
\hline
\end{tabular}

Based on GeneGo modelling, additional candidate genes were tested for association with depression, AUD, and the comorbidity on 9 December, 2007. As in Table 2, queries were qualified by Medical Subject Headings (MeSH) annotation to minimize spurious associations. The actual queries, including $\mathrm{MeSH}$ qualification, are provided as Additional Materials (Tables_2_and_6_Detailed.xls). Column I has a short form of the query and column 2 shows the number of citations that meet the query criteria.

excessive alcohol consumption on folate levels is not new [37] but this analysis puts the environmental effect of alcohol consumption into a genetic context and provides a model for further hypothesis testing.

Candidate gene approaches offer a necessarily limited view of gene-disease association because so much data is missing on virtually all genes. In addition, data mining approaches, if not carefully controlled, can lead to false positive associations. However, by starting with the most reliable data available to select candidate genes, using multiple lines of evidence to test the validity of candidates, then putting the candidates into context, we believe we have developed one valid model of gene-by-environment interaction influencing comorbid depression with AUD. The proposed gene-by-environment interaction model provides a biologically plausible and testable hypothesis on the genetic etiology of comorbid depression with AUD.

With appropriate caveats, this approach to complex disease analysis could be applied to understanding many diseases. Both publication bias towards positive results and bias towards genes that are commonly studied will tend to steer text based evidence in the direction of a relatively limited set of genes. This bias will be countered by the large volumes of data currently being generated without prior hypotheses. In particular, WGA and microarray data are being deposited in publicly available datasets. These data will complement text based approaches by allowing for unbiased tests of the reduced number of hypotheses posed. Indeed, as the volume of data available in databases increases, this type of analysis will become more valuable.

\section{Competing interests}

The authors declare that they have no competing interests.

\section{Authors' contributions}

RCM initially conceived of the work, conducted the database queries as well as the PDG-ACE and GeneGo analyses, and wrote the first draft of the manuscript. BJK developed the PDG-ACE algorithm and helped in subsequent drafting of the manuscript. EFHS put the initial results in the context of one-carbon metabolism and helped with subsequent drafting of the manuscript. MGM participated in the design and coordination of the study and helped draft the final manuscript. All authors read and approved the final manuscript.

\section{Additional material}

\section{Additional file 1}

Detailed PubMed database queries.

Click here for file

[http://www.biomedcentral.com/content/supplementary/1756-

0381-1-2-S1.xls]

\section{Additional file 2}

Manuscript describing the PDG-ACE algorithm.

Click here for file

[http://www.biomedcentral.com/content/supplementary/1756-

0381-1-2-S2.doc]

\section{Additional file 3}

List of 2,531 keywords used in PDG-ACE analysis.

Click here for file

[http://www.biomedcentral.com/content/supplementary/17560381-1-2-S3.txt]

\section{Acknowledgements}

RCM, BJK, and MGM were supported in part by the National Center for Integrative Biomedical Informatics, U54-DA-021519. RCM, EFHS, and MGM were supported in part by the Prechter Bipolar Genetics Fund.

\section{References}

I. Regier DA, Farmer ME, Rae DS, Locke BZ, Keith SJ, Judd LL, Goodwin FK: Comorbidity of mental disorders with alcohol and other drug abuse. Results from the epidemiologic catchment area (ECA) study. Jama 1990, 264(19):25II-2518. 
2. Hasin DS, Glick H: Depressive symptoms and DSM-III-R alcohol dependence: general population results. Addiction 1993, 88(10): $|43|-\mid 436$.

3. Lynskey MT: The comorbidity of alcohol dependence and affective disorders: treatment implications. Drug Alcohol Depend 1998, 52(3):201-209.

4. Wang J, El-Guebaly N: Sociodemographic factors associated with comorbid major depressive episodes and alcohol dependence in the general population. Can J Psychiatry 2004, 49(I):37-44.

5. Goodwin FKJ Kay Redfield: Manic-Depressive Illness. Second edition. New York, NY, Oxford University Press; 2007.

6. Dick DM, Bierut LJ: The genetics of alcohol dependence. Curr Psychiatry Rep 2006, 8(2): 15।-157.

7. Dohrenwend BP, Levav I, Shrout PE, Schwartz S, Naveh G, Link BG, Skodol AE, Stueve A: Socioeconomic status and psychiatric disorders: the causation-selection issue. Science 1992, 255(5047):946-952.

8. Kendler KS, Davis CG, Kessler RC: The familial aggregation of common psychiatric and substance use disorders in the national comorbidity survey: a family history study. $\mathrm{Br} \int P s y-$ chiatry 1997, 170:54I-548.

9. Kendler KS, Heath AC, Neale MC, Kessler RC, Eaves LJ: Alcoholism and major depression in women. A twin study of the causes of comorbidity. Arch Gen Psychiatry 1993, 50(9):690-698.

10. Prescott CA, Aggen SH, Kendler KS: Sex-specific genetic influences on the comorbidity of alcoholism and major depression in a population-based sample of US twins. Arch Gen Psychiatry 2000, 57(8):803-8II.

II. Maglott D, Ostell J, Pruitt KD, Tatusova T: Entrez Gene: genecentered information at NCBI. Nucleic Acids Res 2007, 35(Database issue): $\mathrm{D} 26-31$.

12. Carlson CS, Eberle MA, Kruglyak L, Nickerson DA: Mapping complex disease loci in whole-genome association studies. Nature 2004, 429(6990):446-452.

13. Clark AG, Boerwinkle E, Hixson J, Sing CF: Determinants of the success of whole-genome association testing. Genome Res 2005, I 5(I I): | 1463-1467.

14. Lawrence RW, Evans DM, Cardon LR: Prospects and pitfalls in whole genome association studies. Philos Trans $R$ Soc Lond B Biol Sci 2005, 360( (1460): I 589-I595.

15. Wheeler DL, Barrett T, Benson DA, Bryant SH, Canese K, Chetvernin V, Church DM, Dicuccio M, Edgar R, Federhen S, Feolo M, Geer LY, Helmberg W, Kapustin Y, Khovayko O, Landsman D, Lipman DJ, Madden TL, Maglott DR, Miller V, Ostell J, Pruitt KD, Schuler GD, Shumway M, Sequeira E, Sherry ST, Sirotkin K, Souvorov A, Starchenko G, Tatusov RL, Tatusova TA, Wagner L, Yaschenko E: Database resources of the national center for biotechnology information. Nucleic Acids Res 2007.

16. The Medical subject headings (MeSH) database [http:// www.nlm.nih.gov/mesh/meshhome.html].

17. The database for annotation, visualization and integrated discovery (DAVID) [http://david.abcc.ncifcrf.gov/home.jsp].

18. Ashburner M, Ball CA, Blake JA, Botstein D, Butler H, Cherry JM, Davis AP, Dolinski K, Dwight SS, Eppig JT, Harris MA, Hill DP, IsselTarver L, Kasarskis A, Lewis S, Matese JC, Richardson JE, Ringwald M, Rubin GM, Sherlock G: Gene ontology: tool for the unification of biology. The Gene Ontology Consortium. Nat Genet 2000, 25(I):25-29.

19. Dennis G Jr., Sherman BT, Hosack DA, Yang J, Gao W, Lane HC, Lempicki RA: DAVID: database for annotation, visualization, and integrated discovery. Genome Biol 2003, 4(5): $\mathrm{P3}$.

20. Keller BJ, McEachin RC, Watanabe RM, Mclnnis MG: Identifying hypothetical genetic influences on complex disease phenotypes: San Francisco, California. American Medical Informatics Association; 2008.

21. Hartman JL, Garvik B, Hartwell L: Principles for the buffering of genetic variation. Science 200I, 29 I (5506): I00I-I004.

22. Ekins S, Nikolsky Y, Bugrim A, Kirillov E, Nikolskaya T: Pathway mapping tools for analysis of high content data. Methods Mol Biol 2007, 356:319-350.

23. Ekins S, Bugrim A, Brovold L, Kirillov E, Nikolsky Y, Rakhmatulin E, Sorokina S, Ryabov A, Serebryiskaya T, Melnikov A, Metz J, Nikolskaya $\mathrm{T}$ : Algorithms for network analysis in systems-ADME/
Tox using the MetaCore and MetaDrug platforms. Xenobiotica 2006, 36(I 0-II):877-90I.

24. Acheampong E, Mukhtar M, Parveen Z, Ngoubilly N, Ahmad N, Patel C, Pomerantz RJ: Ethanol strongly potentiates apoptosis induced by HIV-I proteins in primary human brain microvascular endothelial cells. Virology 2002, 304(2):222-234.

25. Kahl KG, Kruse N, Faller H, Weiss H, Rieckmann P: Expression of tumor necrosis factor-alpha and interferon-gamma mRNA in blood cells correlates with depression scores during an acute attack in patients with multiple sclerosis. Psychoneuroendocrinology 2002, 27(6):67I-68I.

26. Le Marchand L, Wilkens LR, Kolonel LN, Henderson BE: The MTHFR C677T polymorphism and colorectal cancer: the multiethnic cohort study. Cancer Epidemiol Biomarkers Prev 2005, 14(5): I 198-I203.

27. Lewis SJ, Lawlor DA, Davey Smith G, Araya R, Timpson N, Day IN, Ebrahim S: The thermolabile variant of MTHFR is associated with depression in the British women's heart and health Study and a meta-analysis. Mol Psychiatry 2006, I I (4):352-360.

28. Song K, Zhao XJ, Marrero L, Oliver P, Nelson S, Kolls JK: Alcohol reversibly disrupts TNF-alpha/TACE interactions in the cell membrane. Respir Res 2005, 6: 123.

29. Tan EC, Chong SA, Lim LC, Chan AO, Teo YY, Tan CH, Mahendran $\mathrm{R}$ : Genetic analysis of the thermolabile methylenetetrahydrofolate reductase variant in schizophrenia and mood disorders. Psychiatr Genet 2004, I4(4):227-23I.

30. Chen PC, DuBois GC, Chen MJ: Mapping the domain(s) critical for the binding of human tumor necrosis factor-alpha to its two receptors. J Biol Chem 1995, 270(6):2874-2878.

31. Natoli G, Costanzo A, Guido F, Moretti F, Levrero M: Apoptotic, non-apoptotic, and anti-apoptotic pathways of tumor necrosis factor signalling. Biochem Pharmacol 1998, 56(8):915-920.

32. Fotin-Mleczek M, Henkler F, Hausser A, Glauner H, Samel D, Graness $A$, Scheurich $P$, Mauri $D$, Wajant $H$ : Tumor necrosis factor receptor-associated factor (TRAF) I regulates CD40-induced TRAF2-mediated NF-kappaB activation. J Biol Chem 2004, 279(I):677-685.

33. Natarajan K, Manna SK, Chaturvedi MM, Aggarwal BB: Protein tyrosine kinase inhibitors block tumor necrosis factor-induced activation of nuclear factor-kappaB, degradation of IkappaBalpha, nuclear translocation of p65, and subsequent gene expression. Arch Biochem Biophys 1998, 352(I):59-70.

34. Liu H, Sidiropoulos P, Song G, Pagliari LJ, Birrer MJ, Stein B, Anrather J, Pope RM: TNF-alpha gene expression in macrophages: regulation by NF-kappa B is independent of c-Jun or C/EBP beta. $J$ Immunol 2000, 164(8):4277-4285

35. Moat SJ, Ashfield-Watt PA, Powers HJ, Newcombe RG, McDowell IF: Effect of riboflavin status on the homocysteine-lowering effect of folate in relation to the MTHFR (C677T) genotype. Clin Chem 2003, 49(2):295-302.

36. Pickell L, Tran P, Leclerc D, Hiscott J, Rozen R: Regulatory studies of murine methylenetetrahydrofolate reductase reveal two major promoters and NF-kappaB sensitivity. Biochim Biophys Acta 2005, I73 I(2): 104-II4.

37. Mason JB, Choi SW: Effects of alcohol on folate metabolism: implications for carcinogenesis. Alcohol 2005, 35(3):235-24I.

38. Mischoulon D, Raab MF: The role of folate in depression and dementia. J Clin Psychiatry 2007, 68 Suppl 10:28-33.

39. Coppen A, Abou-Saleh MT: Plasma folate and affective morbidity during long-term lithium therapy. $\mathrm{Br} J$ Psychiatry 1982, | 41:87-89.

40. Coppen A, Swade C, Jones SA, Armstrong RA, Blair JA, Leeming RJ: Depression and tetrahydrobiopterin: the folate connection. J Affect Disord 1989, 16(2-3): 103-107.

4I. Folstein M, Liu T, Peter I, Buell J, Arsenault L, Scott T, Qiu WW: The homocysteine hypothesis of depression. Am J Psychiatry 2007, 164(6):86I-867.

42. Abou-Saleh MT, Coppen A: The biology of folate in depression: implications for nutritional hypotheses of the psychoses. Psychiatr Res 1986, 20(2):91-101.

43. Abou-Saleh MT, Coppen A: Serum and red blood cell folate in depression. Acta Psychiatr Scand 1989, 80(1):78-82.

44. Abou-Saleh MT, Coppen A: Folic acid and the treatment of depression. J Psychosom Res 2006, 61 (3):285-287. 
45. Gilbody S, Lewis S, Lightfoot T: Methylenetetrahydrofolate reductase (MTHFR) genetic polymorphisms and psychiatric disorders: a HuGE review. Am J Epidemiol 2007, 165(I): I-I3.

46. McGuffin P, Knight J, Breen G, Brewster S, Boyd PR, Craddock N, Gill M, Korszun A, Maier W, Middleton L, Mors O, Owen MJ, Perry J, Preisig M, Reich T, Rice J, Rietschel M, Jones L, Sham P, Farmer AE: Whole genome linkage scan of recurrent depressive disorder from the depression network study. Hum Mol Genet 2005, I 4(22):3337-3345.

47. Demirhan O, Tastemir D: Cytogenetic effects of ethanol on chronic alcohol users. Alcohol Alcohol 2008, 43(2): 127-I36.

48. Coppen A, Bailey !: Enhancement of the antidepressant action of fluoxetine by folic acid: a randomised, placebo controlled trial. J Affect Disord 2000, 60(2): $121-130$.

49. Coppen A, Bolander-Gouaille C: Treatment of depression: time to consider folic acid and vitamin B I2. J Psychopharmacol 2005, 1 9(I):59-65.

50. Coppen A, Chaudhry S, Swade C: Folic acid enhances lithium prophylaxis. J Affect Disord 1986, I0(1):9-13.

51. Black JL 3rd, O'Kane DJ, Mrazek DA: The impact of CYP allelic variation on antidepressant metabolism: a review. Expert Opin Drug Metab Toxicol 2007, 3(1):2I-3I.

52. Camilleri M: Pharmacogenomics and serotonergic agents: research observations and potential clinical practice implications. Neurogastroenterol Motil 2007, 19 Suppl 2:40-45.

53. McAlpine DE, O'Kane DJ, Black JL, Mrazek DA: Cytochrome P450 2D6 genotype variation and venlafaxine dosage. Mayo Clin Proc 2007, 82(9): $1065-1068$.

54. Pestka EL, Hale AM, Johnson BL, Lee JL, Poppe KA: Cytochrome P450 testing for better psychiatric care. J Psychosoc Nurs Ment Health Serv 2007, 45(10): 15-18.

Publish with Bio Med Central and every scientist can read your work free of charge

"BioMed Central will be the most significant development for disseminating the results of biomedical research in our lifetime. "

Sir Paul Nurse, Cancer Research UK

Your research papers will be:

- available free of charge to the entire biomedical community

- peer reviewed and published immediately upon acceptance

- cited in PubMed and archived on PubMed Central

- yours - you keep the copyright
BioMedcentral 